\title{
Metamagnetism in hydrophobically induced carboxylate (phenylmalonate)-bridged copper(II) layers
}

\author{
Jorge Pasán, ${ }^{a}$ Joaquín Sanchiz, ${ }^{b}$ Catalina Ruiz-Pérez, ${ }^{* a}$ Javier Campo, ${ }^{c}$ Francesc Lloret $^{d}$ and Miguel Julve ${ }^{d}$ \\ Received (in Cambridge, UK) 14th February 2006, Accepted 22nd May 2006 \\ First published as an Advance Article on the web 14th June 2006 \\ DOI: $10.1039 / b 602144 a$
}

Self-assembly of copper(II) ions, phenylmalonate and pyrimidine yields the layered compound $[\mathrm{Cu}(\mathrm{pym})(\mathrm{Phmal})]_{n}$ (1) where intralayer ferro- and interlayer antiferromagnetic interactions occur with three-dimensional antiferromagnetic ordering at $T_{\mathrm{c}}=2.15 \mathrm{~K}$.

The construction of multidimensional magnetic materials with magnetic ordering is one of the major challenges in magnetochemistry. ${ }^{1}$ For such a purpose, the construction of three-dimensional (3D) systems is a good approach, but a deep study of the nature and intensity of the coupling among the paramagnetic centres in these systems is precluded due to the lack of theoretical models to analyze the magnetic ordered state. On the other hand, interesting studies have been performed on 2D systems with different intraand interlayer magnetic interactions., ${ }^{2,3}$ Previous studies by Kahn and co-workers in designing molecule-based magnets have shown that organizing ferromagnetic arrangements in extended $2 \mathrm{D}$ or $3 \mathrm{D}$ systems is quite a difficult task. ${ }^{1}$ The choice of a specifically tailored building block is crucial in the design of systems with tunable dimensionality displaying ferromagnetic coupling.

The malonate-bridged copper(II) complexes show a wide variety of molecular architectures which exhibit ferromagnetic coupling in most cases. ${ }^{4-8}$ In previous studies with these compounds, we have observed that the insertion of additional groups in the malonate skeleton (for instance, a phenyl group) modifies the supramolecular interactions and the dimensionality of the coordination polymer, but the ferromagnetic nature of the magnetic coupling is kept as in the compound $\left[\mathrm{Cu}\left(\mathrm{H}_{2} \mathrm{O}\right)_{3}\right]\left[\mathrm{Cu}(\mathrm{Phmal})_{2}\right]\left(\mathrm{H}_{2} \mathrm{Phmal}=\right.$ phenylmalonic acid). ${ }^{9}$ The use of potentially bridging nitrogen donor heterocycles as coligands has proved to be very appealing because of the construction of supramolecular motifs with varied functions. ${ }^{10-13}$ In the present work, we focus on the structure and variable-temperature magnetic study of the layered compound $[\mathrm{Cu}(\text { pym })(\mathrm{Phmal})]_{n}(\mathbf{1})($ pym $=$ pyrimidine $)$ together with a brief analysis of the supramolecular effects that determine its twodimensional structure. The compound has a metamagnetic behaviour due to the coexistence of intralayer ferromagnetic and

${ }^{a}$ Laboratorio de Rayos X y Materiales Moleculares, Dpto. de Física Fundamental II, Universidad de La Laguna, Avda. Astrofisico Francisco Sánchez s/n,La Laguna, Spain.E-mail: caruiz@ull.es;

Fax: +34922 3183 20; Tel: +34922318300

${ }^{b}$ Laboratorio de Rayos X y Materiales Moleculares, Dpto. de Química Inorgánica, Universidad de La Laguna, La Laguna, Spain

${ }^{c}$ Instituto de Ciencia de Materiales de Aragón, CSIC-Universidad de Zaragoza, 50009 Zaragoza, Spain

${ }^{d}$ Departament de Química InorgànicalInstituto de Ciencia Molecular, Facultat de Química, Universitat de València, Av. Dr. Moliner 50, 46100 Burjassot (València), Spain interlayer antiferromagnetic interactions, the critical magnetic field being $H_{\mathrm{c}}=130 \mathrm{G}$.

The structure of $\mathbf{1}$ consists of a sheet-like arrangement of (pym)copper(II) units bridged by carboxylate-phenylmalonate groups growing in the $a b$ plane. $\dagger$ Each copper atom is linked to four other symmetry-related copper atoms (Fig. 1) through equatorial-equatorial anti-syn carboxylate bridges [the coppercopper separation is 5.121(17) $\mathrm{A}$ ]. A corrugated square grid of copper atoms results, the monodentate pym ligand of adjacent metal atoms being located above and below each layer. The pym and phenyl groups of each $[\mathrm{Cu}(\mathrm{pym})(\mathrm{Phmal})]$ unit are in the transposition. The neutral sheets are stacked along the $c$ axis, the shortest interlayer copper-copper separation being 13.45(4) $\AA$ (Fig. 2). Weak interactions between hydrophobic groups of adjacent layers in $\mathbf{1}$ are the origin of its 2D structure.

Each copper atom exhibits a square-pyramidal environment ( $\tau$ value ${ }^{14}$ being 0.02 ) with four carboxylate-phenylmalonate oxygen atoms $[\mathrm{O}(1), \mathrm{O}(2), \mathrm{O}(3)$ and $\mathrm{O}(4)]$ from three different Phmal ligands building the basal plane [mean value for the $\mathrm{Cu}-\mathrm{O}$ bond distance is $1.960(6) \AA$ ] while a nitrogen atom from the pym ligand $[\mathrm{N}(1)]$ occupies the axial position $[\mathrm{Cu}(1)-\mathrm{N}(1)=2.252(3) \AA]$. The copper atom is shifted by 0.2049 (10) $\AA$ from the mean basal plane towards the apical position.

The square grid of copper(II) ions occurring in $\mathbf{1}$ is analogous to those observed for the malonate complexes of cobalt(II) and zinc(II) ions in the presence of $\mathrm{N}$-donor heterocycles as coligands. ${ }^{15}$

The temperature dependence of the $\chi_{\mathrm{M}} T$ product $\left[\chi_{\mathrm{M}}\right.$ being the magnetic susceptibility per copper(II) ion] in the temperature range $1.8-300 \mathrm{~K}$ is shown in Fig. $3 .+\chi_{\mathrm{M}} T$ at room temperature is $0.42 \mathrm{~cm}^{3} \mathrm{~mol}^{-1} \mathrm{~K}$, a value which is as expected for a magnetically isolated spin doublet. Upon cooling, $\chi_{\mathrm{M}} T$ continuously increases to reach a value of $25.9 \mathrm{~cm}^{3} \mathrm{~mol}^{-1} \mathrm{~K}$ at $2.15 \mathrm{~K}$, and further decreases to $17.5 \mathrm{~cm}^{3} \mathrm{~mol}^{-1} \mathrm{~K}$ at $1.8 \mathrm{~K}$. A susceptibility maximum

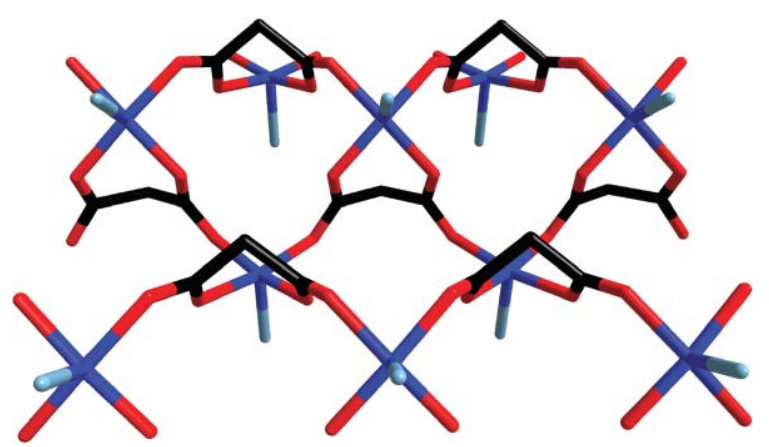

Fig. 1 Central projection of the formation of the square grid of copper(II) ions through the carboxylate-phenylmalonate linkage. 


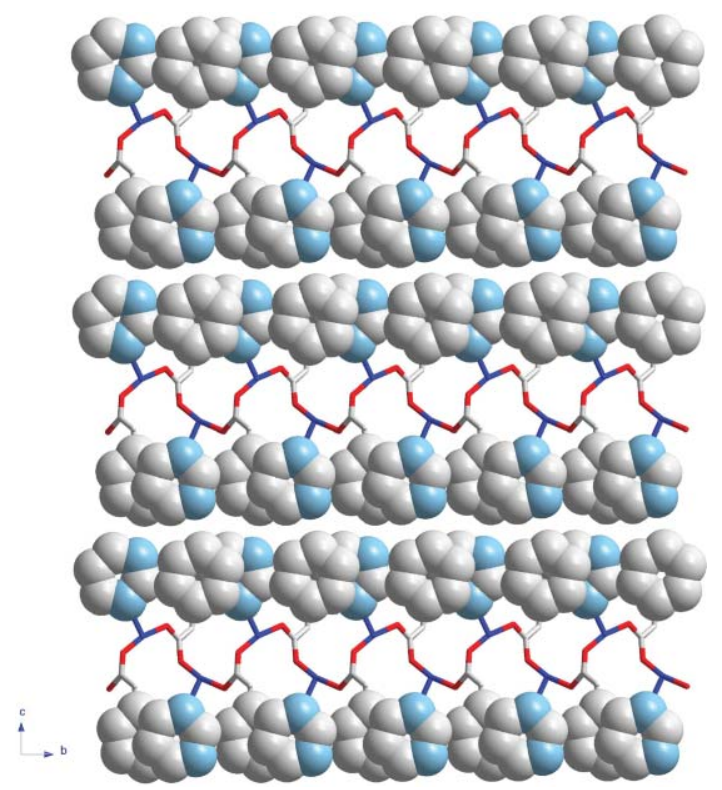

Fig. 2 Projection of the crystal packing of 1 through the $a$ axis.

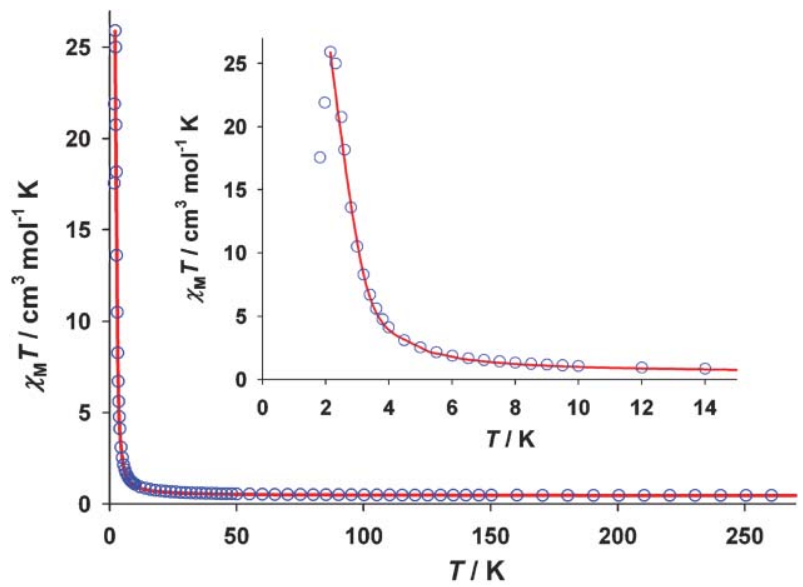

Fig. $3 \chi_{\mathrm{M}} T$ vs. $T$ plot for 1 under applied magnetic fields of $1 \mathrm{~T}(T \geqslant$ $12 \mathrm{~K})$ and $100 \mathrm{G}(T<12 \mathrm{~K})$ : $(\bigcirc)$ experimental data; $(-)$ best-fit curve through eqn. (1) (see text). The inset shows a detail of the low temperature region.

is observed at $2.15 \mathrm{~K}$ under $H \leqslant 130 \mathrm{G}$. This maximum disappears for $H>130 \mathrm{G}$ (Fig. 4). These features correspond to a metamagnetic-like behaviour which is due to the coexistence of ferro- and antiferromagnetic interactions, the latter being overcome by an applied field larger than $130 \mathrm{G}$. The magnetization (M) versus $H$ plot at $1.8 \mathrm{~K}$ confirms this metamagnetic behaviour (Fig. 5). The abrupt increase of $M$ at low fields and the saturation value of $1.06 \mathrm{BM}$ for $H>3 \mathrm{~T}$ evidence the presence of a ferromagnetic interaction between the spin doublets. The sigmoidal shape of the magnetization plot at very low fields (see inset of Fig. 5) with a value for the critical field $\left(H_{\mathrm{c}}\right)$ of $130 \mathrm{G}$ accounts for a weak antiferromagnetic interaction $\left(\mathrm{ca} .10^{-2} \mathrm{~cm}^{-1}\right)$.

The susceptibility maximum at $H<H_{\mathrm{c}}$ corresponds to the onset of a long range antiferromagnetic ordering. The value of the ordering temperature $\left(T_{\mathrm{c}}=2.15 \mathrm{~K}\right)$ is determined by the position of the maximum of the out-of-phase signal $\left(\chi_{\mathrm{M}}{ }^{\prime \prime}\right)$ (see Fig. 6).

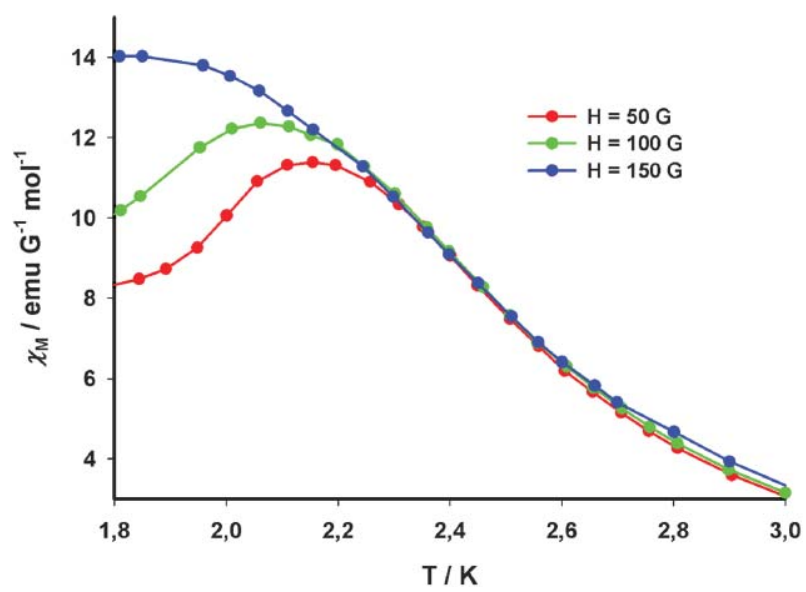

Fig. $4 \chi_{\mathrm{M}}$ vs. $T$ plot for $\mathbf{1}$ at different applied magnetic fields.

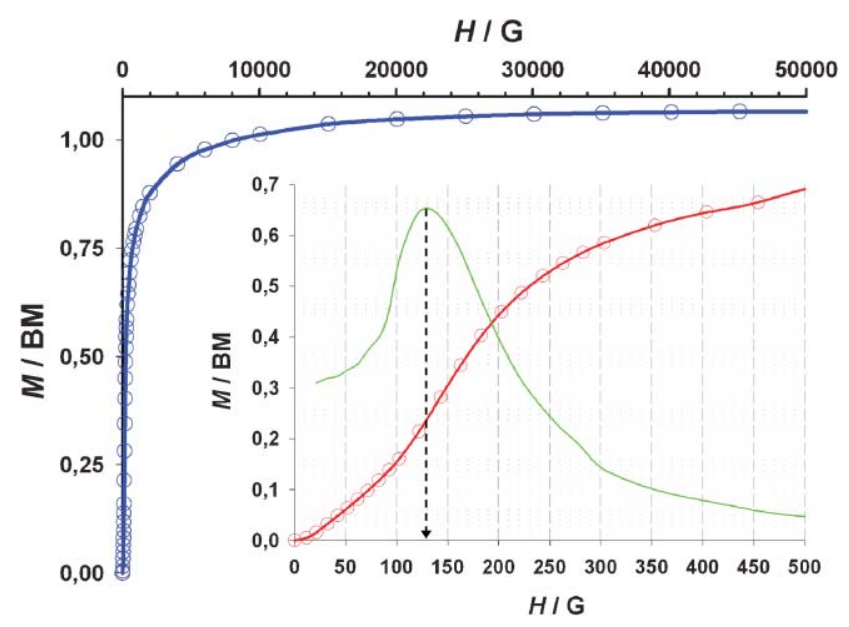

Fig. 5 Magnetization vs. $H$ plot at $1.8 \mathrm{~K}$. The inset shows the sigmoidal shape of the magnetization in the low field region together with the $\partial M / \partial H$ (in green).

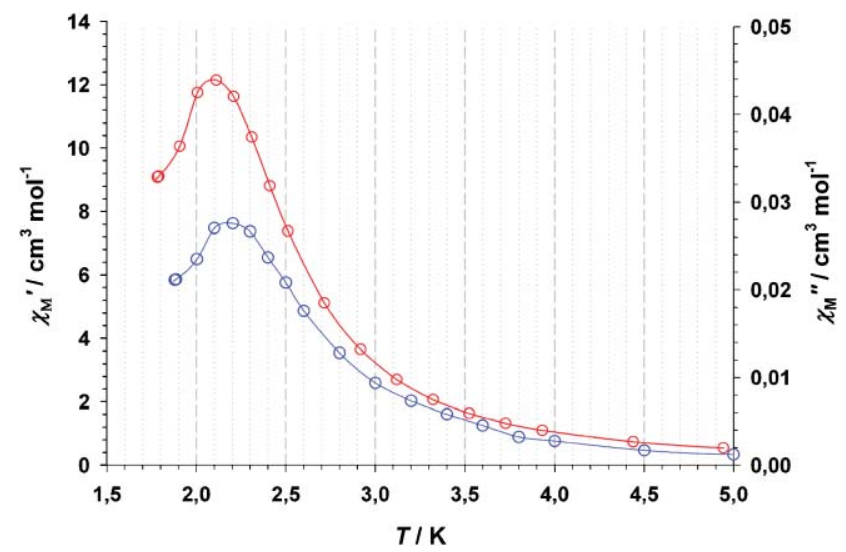

Fig. 6 In-phase (red) and out-of-phase (blue) ac signals of $\mathbf{1}$ in a $1 \mathrm{G}$ field oscillating at $330 \mathrm{~Hz}$ without dc magnetic field: $(\bigcirc, \bigcirc)$ experimental data; (-) eye-guide line.

In the light of the above magneto-structural data of $\mathbf{1}$, its magnetic behaviour would correspond to that of a ferromagnetically coupled square grid of copper(II) ions through a carboxylate-bridge in the anti-syn conformation, each layer 
interacting antiferromagnetically with the adjacent ones. Consequently, the high temperature magnetic data of $\mathbf{1}$ were analyzed through the expression of the magnetic susceptibility by Baker et al. derived from the high-temperature expansion series for an isotropic ferromagnetic square lattice with interacting spin doublets. ${ }^{16}$ The spin Hamiltonian is defined as $\hat{H}=-J \Sigma_{\mathrm{i}} \hat{S}_{\mathrm{i}} \cdot \hat{S}_{\mathrm{i}+1}$ and the series takes the form defined by eqn. (1)

$$
\chi_{\mathrm{M}}=\left(\frac{N \beta^{2} g^{2}}{3 k_{\mathrm{B}} T} S(S+1)\right)\left[1+\sum_{n \geq 1} a_{n} \frac{x^{n}}{2^{n}}\right]
$$

where $N, g, \beta$ and $k_{\mathrm{B}}$ have their usual meaning, $x=J / k_{\mathrm{B}} T, J$ is the intralayer magnetic coupling and $a_{n}$ are coefficients which run up to $n=10$. Least-squares fit of the experimental data to eqn. (1) in the temperature range $5-300 \mathrm{~K}$ leads to $J=+5.6(1) \mathrm{cm}^{-1}, g=$ 2.12(2) and $R=8.9 \times 10^{-5}$ ( $R$ is the agreement factor defined as $\left.\Sigma_{\mathrm{i}}\left[\left(\chi_{\mathrm{M}} T\right)_{\text {obs }}(\mathrm{i})-\left(\chi_{\mathrm{M}} T\right)_{\text {calc }}(\mathrm{i})\right]^{2} / \Sigma\left[\left(\chi_{\mathrm{M}} T\right)_{\text {obs }}(\mathrm{i})\right]^{2}\right)$. The calculated curve matches very well the experimental data in the 5-300 K temperature range. The value of $J$ for $\mathbf{1}$ is the largest one observed in the family of carboxylate(phenylmalonate)-bridged copper(II) complexes $\left(J \text { ranging from }-0.59(1) \text { to }+4.44(5) \mathrm{cm}^{-1}\right)^{9,17}$ and it is within the range of previously reported magnetic couplings of antisyn carboxylate(malonate)-bridged copper(II) complexes. ${ }^{4}$ The smaller value of $\tau$ of the copper atom in the case of $\mathbf{1}$ when compared to other examples with the phenylmalonate ligand reduces the admixture of the $\mathrm{d}_{z^{2}}$ character in the $\mathrm{d}_{x^{2}-y^{2}}$ magnetic orbital and reinforces the magnetic coupling through the equatorial-equatorial exchange pathway, everything being similar.

The weak antiferromagnetic interaction $\left(c a .10^{-2} \mathrm{~cm}^{-1}\right)$ between the ferromagnetically coupled layers of $\mathbf{1}$ leads to a 3D antiferromagnetic ordering with a $T_{\mathrm{c}}$ value of $2.15 \mathrm{~K}$ and accounts for the observed metamagnetic behaviour of this compound. The small value for the interlayer magnetic interaction is in agreement with the large interlayer metal-metal separation and most likely it is dipolar through-space in origin, given the lack of hydrogenbonds and $\pi$-type interactions between the layers. This situation is similar to that observed in layered compounds of formula $\mathrm{M}_{2}(\mathrm{OH})_{3} \mathrm{X}[\mathrm{M}=\mathrm{Cu}(\mathrm{II})$ and $\mathrm{Co}(\mathrm{II}) ; \mathrm{X}=$ organic anion] which exhibit dominant ferromagnetic in-plane interactions, and ferro- or antiferromagnetic inter-plane interactions depending on the interlayer separation. These studies have shown that the large spacings favour spontaneous magnetization. ${ }^{18}$

We can conclude that the introduction of the phenyl group in the malonate carbon skeleton and the presence of coordinated pyrimidine ligand lead to the formation of a hydrophobic layer in which both groups are involved. This aggregation allows the formation of the square grid sheet of carboxylate-bridged copper(II) ions. The analysis of the magnetic properties of $\mathbf{1}$ shows the occurrence of a metamagnetic behaviour with magnetic ordering below $2.15 \mathrm{~K}$, which arises from the coexistence of intralayer ferro- and interlayer antiferromagnetic interactions.

Further work is planned concerning the preparation of phenylmalonate-copper(II) complexes with pym-like ligands in order to tune the interlayer separation and to correlate it with the nature and magnitude of the interlayer magnetic coupling.

We thank the Ministerio Español de Educación y Ciencia for funding through projects MAT2004-03112 and CTQ2004-03633 and J.P. is grateful for a predoctoral fellowship, AP2001-3322.

\section{Notes and references}

$\uparrow$ Synthesis of 1: A methanolic solution $\left(3 \mathrm{~cm}^{3}\right)$ of pyrimidine $(0.5 \mathrm{mmol}$, $40 \mathrm{mg}$ ) was added dropwise to an aqueous solution $\left(5 \mathrm{~cm}^{3}\right)$ of phenylmalonatecopper(II) $(0.5 \mathrm{mmol}, 120 \mathrm{mg})$ prepared as previously described. ${ }^{9}$ Poor quality blue plate-like crystals suitable for X-ray diffraction were obtained from the resulting pale blue solution after a few days by slow evaporation at room temperature. Crystal data of $\mathbf{1}$ : the data collection was carried out on a Bruker-Nonius KappaCCD diffractometer at $293 \mathrm{~K}$, using graphite-monochromated Mo-K $\alpha$ radiation $(\lambda=0.71073 \AA)$ by a $\varphi-\omega$ scan method. The structure was solved by direct methods and refined by least squares procedures on $F^{2}$ using SHELX-97 (G. M. Sheldrick, program for the solution and refinement of crystal structures, University of Göttingen, Germany, 1997) incorporated into the WINGX software package (L. J. Farrugia, J. Appl. Crystallogr., 1999, 32, 659). Crystal dimensions $0.75 \times 0.30 \times 0.05 \mathrm{~mm} ; T=293(2) \mathrm{K}$; monoclinic; $P 2_{1} ; a=6.4460(16), b=6.4460(12), c=14.444(3) \AA$ and $\beta=$ $98.145(12)^{\circ} ; V=594.1(2) \AA^{3}, Z=2$; Flack parameter $=0.57(17) ; \rho_{\text {calc }}=$ $1.799 \mathrm{~g} \mathrm{~cm}^{-3}, 2 \theta_{\max }=31.94^{\circ} ; 3279$ reflections measured of which 1980 are unique; $R_{1}=0.1253, w R_{2}=0.3153$ for $I>2 \sigma(I)$; residual electron density 7.946 and $-1.710 \mathrm{e}^{-3}$. These high peaks are located at $c a .2 .6 \AA$ from the copper atoms and can be ascribed to unresolved twinning effects. All nonhydrogen atoms were refined anisotropically. Hydrogen atoms are placed geometrically and refined as a riding model. CCDC 297276. For crystallographic data in CIF or other electronic format see DOI: $10.1039 / \mathrm{b} 602144 \mathrm{a}$

$\$$ Dc and ac magnetic susceptibility measurements of 1 were performed on polycrystalline samples with a Quantum Design SQUID magnetometer. Corrections for the diamagnetism of the constituent atoms and the sample holder were applied.

1 O. Kahn, Molecular Magnetism, VCH, New York, 1993 and references therein.

2 V. Laget, C. Hornick, P. Rabu, M. Drillon and R. Ziessel, Coord. Chem. Rev., 1998, 180, 1533.

3 M. Drillon, P. Panissod, P. Rabu, J. Souletie, V. Ksenofontov and P. Gutlich, Phys. Rev. B, 2002, 6510, 4404.

4 J. Pasán, F. S. Delgado, Y. Rodríguez-Martín, M. Hernández-Molina, C. Ruiz-Pérez, J. Sanchiz, F. Lloret and M. Julve, Polyhedron, 2003, 22, 2143.

5 C. Ruiz-Pérez, Y. Rodríguez-Martín, M. Hernández-Molina, F. S. Delgado, J. Pasán, J. Sanchiz, F. Lloret and M. Julve, Polyhedron, 2003, 22, 2111; J. Sanchiz, Y. Rodriguez-Martin, C. RuizPerez, A. Mederos, F. Lloret and M. Julve, New J. Chem., 2002, 26, 1624.

6 I. G. de Muro, F. A. Mautner, M. Insausti, L. Lezama, M. I. Arriortua and T. Rojo, Inorg. Chem., 1998, 37, 3243.

7 F. S. Delgado, J. Sanchiz, C. Ruiz-Pérez, F. Lloret and M. Julve, Inorg. Chem., 2003, 42, 5938.

8 T. F. Liu, H. L. Sun, S. Gao, S. W. Zhang and T. C. Lau, Inorg. Chem., 2003, 42, 4792.

9 J. Pasán, J. Sanchiz, C. Ruiz-Pérez, F. Lloret and M. Julve, New J. Chem., 2003, 27, 1557.

10 J. M. Lehn, Supramolecular Chemistry: Concepts and Perspectives, VCH, Weinheim, 1995.

11 C. Kaes, A. Katz and M. W. Hosseini, Chem. Rev., 2000, 100, 3553.

12 M. Fujita, Chem. Soc. Rev., 1998, 27, 417

13 D. L. Caulder and K. N. Raymond, Acc. Chem. Res., 1999, 32, 975.

14 A. W. Addison, T. N. Rao, J. Reedijk, J. van Rijn and G. C. Verschoor, J. Chem. Soc., Dalton Trans., 1984, 1349.

15 F. S. Delgado, J. Sanchiz, C. Ruiz-Pérez, F. Lloret and M. Julve, CrystEngComm, 2003, 5, 48, 280.

16 G. S. Rushbrooke, G. A. Baker and P. J. Wood, Phase Transitions and Critical Phenomena, vol. III, ed. C. Domb and M. S. Green, Academic Press, 1974.

17 J. Pasán, J. Sanchiz, C. Ruiz-Pérez, F. Lloret and M. Julve, Eur. J Inorg. Chem., 2004, 4081; J. Pasán, J. Sanchiz, C. Ruiz-Pérez, F. Lloret and M. Julve, Inorg. Chem., 2005, 44, 7794.

18 V. Laget, C. Hornick, P. Rabu, M. Drillon and R. Ziessel, Coord. Chem. Rev., 1998, 178-180, 1533. 